\title{
Computational Fluid Dynamics Modeling of Extrathoracic Airway Flush: Evaluation of High Flow Nasal Cannula Design Elements \\ Thomas L Miller ${ }^{1,2 *}$, Babak Saberi ${ }^{3}$ and Shadi Saberi ${ }^{3}$
}

${ }^{1}$ Vapotherm Inc., Exeter, New Hampshire, USA

${ }^{2}$ Sidney Kimmel Medical College, Thomas Jefferson University, Philadelphia, USA

${ }^{3}$ Green Twirl Energy, Ontario, Canada

\begin{abstract}
Objective: High flow nasal cannula (HFNC) is an evolving respiratory therapy whereby high flow rates of conditioned breathing gas are delivered into the nasal cavity to purge anatomical dead space of $\mathrm{CO}_{2}$ rich expired gas. The aim of this project was to create a computational fluid dynamics (CFD) model to evaluate the fluid patterns in the human nasal and pharyngeal cavities with HFNC application, and quantify time to purge for two cannula configurations.

Methods: Three-dimensional geometry of the human airway was used to define the extrathoracic dead space and the two cannula designs tested incorporate large vs small bore nasal prong configurations (Vapotherm, Exeter, $\mathrm{NH}$, USA). The fluid flow simulations were performed using FLOW-3D software, set up for a cannula flow rate of $20 \mathrm{~L} \cdot \mathrm{min}^{-1}$ and run until steady state.

Results: Basic flow patterns were similar between cannulae, creating vortices around a central inward flow path Flow velocity around the vortices was greater with the small prong cannula, resulting in a lower pressure in each region of the nasal and nasopharyngeal space. The calculation of purge time revealed that the small prong nasal cannula was able to clear the nasal, pharyngeal and oral cavities in 2.2 seconds, whereas the large bore cannula required 3.6 seconds (64\% longer).

Conclusion: The current CFD data validate that a smaller bore nasal prong facilitates the purge action, which is related to velocity and dynamic energy induced by the tighter prong nozzle as opposed to the lesser occlusion of the nares.
\end{abstract}

Keywords: High flow therapy; High velocity nasal insufflation; Respiratory support; Non-invasive ventilation; Nasal gas insufflation; Computational fluid dynamics; Airway modeling

\section{Introduction}

High flow nasal cannula (HFNC) is an evolving respiratory therapy modality based on delivering high flow rates of humidified respiratory gas to a patient's nasal cavity through a nasal cannula. These high flow rates were demonstrated to improve breathing efficiency by purging anatomical extrathoracic dead space of expired gas, and creating anatomical reservoir of enriched inspiratory gas [1,2]. With proper conditioning of the delivered gas to near $100 \%$ relative humidity and body temperature, the administration of these high flow rates is accomplished without drying and subsequently damaging the nasal tissues [3-5]. Animal studies indicate that cannula design influences the physiologic impact [6], but little is known about the fluid movement patterns in the human nasal cavity and pharyngeal space with HFNC application.

Evidence suggests that the mechanisms of action for HFNC include purging nasal, pharyngeal and oral dead space (extrathoracic dead space) [7], reducing inspiratory resistance, improving conductance and pulmonary compliance [8], providing mild positive distending pressure [9-11] and restoring mucocilliary function through rehydration $[12,13]$. By reducing dead space, we can reduce the minute ventilation $\left(\mathrm{V}_{\mathrm{E}}\right)$ needed to accomplish adequate alveolar ventilation $\left(\mathrm{V}_{\mathrm{A}}\right)$ and therefore reduce work of breathing. Dead space elimination tactics have been used for years in the form of tracheal gas insufflation $[14,15]$ and transtracheal oxygen delivery [16], which rely on tubes placed in the trachea and are therefore more invasive. In recent years, advancements in heated humidification devices have made it possible to accomplish ventilation by way of dead space elimination using a non-invasive nasal cannula.
Our preclinical physiologic research demonstrated that a less occlusive prong design provided for better physiologic efficacy, and did so at lower flow rates with less resulting airway pressure development. Whereas these data do confirm the hypothesis that the principle mechanism of action in high flow nasal insufflation is purging anatomical dead space, these results also indicate that cannula design may play an important role. Among other points of value in understanding cannula design, the effects of cannula prong size in terms of occlusion of the nares is important relative to differentiating HFNC delivery system design specifications.

The goal of the current study was to create a computational fluid dynamics (CFD) model to better understand the dynamics of nasal cannula delivered respiratory gas flow through the nasal cavity and pharyngeal space. Furthermore, we aimed to evaluate two cannula designs that incorporate large $v s$ small bore nasal prong configurations to determine which would be optimal for utilizing HFNC as a ventilatory support modality. We hypothesized that the small bore nasal prong cannula would lead to a more rapid flush of the extrathoracic airway.

*Corresponding author: Thomas L Miller, Clinical and Scientific Affairs, 22 Industrial Drive, Suite 1 Exeter, NH-03833, USA, Tel: (856) 279-1227; Fax: (603) 658-0181; E-mail: tmiller@vtherm.com

Received September 22, 2016; Accepted October 26, 2016; Published October 31,2016

Citation: Miller TL, Saberi B, Saberi S (2016) Computational Fluid Dynamics Modeling of Extrathoracic Airway Flush: Evaluation of High Flow Nasal Cannula Design Elements. J Pulm Respir Med 6: 376. doi: 10.4172/2161-105X.1000376

Copyright: @ 2016 Miller TL, et al. This is an open-access article distributed under the terms of the Creative Commons Attribution License, which permits unrestricted use, distribution, and reproduction in any medium, provided the original author and source are credited. 


\section{Methods}

The CFD method was used as a powerful numerical technique for the study of flow patterns in systems with complex geometries and those in which measurements cannot easily be carried out. In performing the CFD analysis, the first step was to create a realistic representation of the physical geometry of the system, and then discretized it to small elements that, in their entirety, form the computational domain or "mesh". In the next steps, suitable mathematical equations describing the nature of the flow were selected and applied to each mesh element. The boundaries of the system are defined, and the flow conditions at the inlets and outlets are specified. A state of the art CFD software package (FLOW-3D, Flow Science Inc., Santa Fe, NM, USA) was used to provide a selection of computer-aided design (CAD) and meshing features, along with a model library corresponding to various flow characteristics, and optimized numerical solvers that provide accurate solutions.

\section{Study design and flow conditions}

The three-dimensional geometry of the human airway (Figure 1A) was obtained in stereolithography (STL) format from Flow Science, and was transferred to FLOW-3D'. The geometry STL file was that of a small adult based on anatomical size references and captured all germane anatomical features, including the nasal concha associated with the lateral walls, with the exception of an incomplete nasal septum. The anatomical feature of a nasal septum was added to the geometry for accuracy, and was consistent with accepted anatomical references. A box representing the outside environment was attached to the geometry so that there would be no outside influence on the flow dynamics that were calculated to occur within the nasal and oral anatomy.

The simulation was set up for a flow rate of $20 \mathrm{~L} \cdot \mathrm{min}^{-1}$ and was run until a steady state solution was obtained. The flow rate of $20 \mathrm{~L} \cdot \mathrm{min}^{-1}$ was chosen because the geometry was related to a small adult, wherein 20 $\mathrm{L} \cdot \mathrm{min}^{-1}$ is on the low end of appropriate flow rates for HFNC. Therefore, choosing this flow rate would insure that the purging effect was not saturated, thus limiting the ability of the model to detect an influence of cannula design. A statistical analysis was performed on the results to obtain purge time (flow residence time in the nasopharyngeal volume).
Two nasal cannula configurations were tested, as shown in Figure $1 \mathrm{~B}$ and $1 \mathrm{C}$. The small bore nasal cannula design had a nasal prong diameter of 0.170 in $(4.318 \mathrm{~mm})$ at the tip, which determined the radius of the gas nozzle, and 0.203 in $(5.161 \mathrm{~mm})$ at the base which influences the degree to which the cannula occluded/obstructed the nasal openings (nares). The large bore cannula design has a diameter of 0.223 in $(5.668 \mathrm{~mm})$ at the tip and 0.305 in $(7.747 \mathrm{~mm})$ at the base. It should be noted that the small bore design was configured to deliver flow to the nasal prongs from two directions via two connecting tubes which converged at a small diameter reservoir from which the prongs emerged, while the large bore cannula was configured to deliver flow to the nasal prongs from one direction using a larger diameter connecting tube.

\section{Flow $3 D^{\circ}$ model parameters}

A low Mach number single fluid model was defined in FLOW-3D. This is a weakly-compressible fluid model wherein full compressibility in the equations of motion is not considered and does not capture acoustic phenomena that happen at high Mach numbers (above 0.3). However, the dependence of gas density to pressure according to the ideal gas law is considered.

A standard k-epsilon model was used to describe turbulence. A second order discretization was applied as advection scheme. The units for length, mass and time were inches, grams and seconds, respectively. The simulation was run with double precision option to ensure a higher level of numerical accuracy.

\section{CFD methodology}

The fluid flow simulations were performed using FLOW-3D generalpurpose high fidelity CFD software. The geometry was imported in FLOW-3D as a stereolithography (STL) model. A computational multiblock mesh was constructed using 6 blocks and a total of 2,480,500 grid cells. FLOW-3D incorporates a special technique, known as the FAVOR $^{\text {ix }}$ (fractional area volume obstacle representation) method, which is used to define general geometric regions within the rectangular grid. The philosophy behind FAVOR ${ }^{\mathrm{m}}$ is that numerical algorithms are
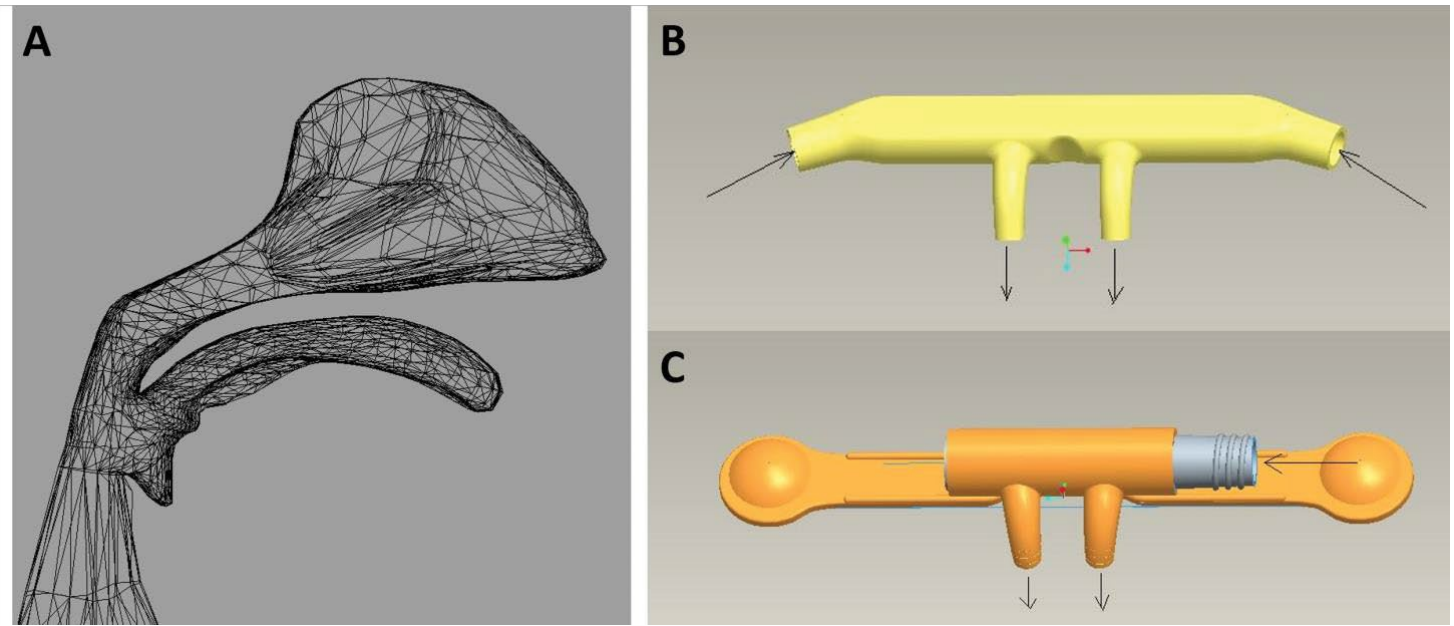

Figure 1: Anatomical and Nasal Cannula Geometry. Three-dimensional geometry of the human airway (A) was obtained as stereolithography model for the fluid flow simulations. Nasal cannula geometry was taken from computer aided design (CAD) files for the two cannula configurations being evaluated. The small bore nasal prong design $(B)$ had a prong diameter of $0.170 \mathrm{in}(4.318 \mathrm{~mm})$ at the tip, which determined the radius of the gas nozzle, and $0.203 \mathrm{in}(5.161 \mathrm{~mm})$ at the base which influences the degree to which the cannula occluded/obstructed the nasal openings (nares). The large bore cannula design (C) had a diameter of 0.223 in (5.668 $\mathrm{mm})$ at the tip and $0.305 \mathrm{in}(7.747 \mathrm{~mm})$ at the base. The small bore design was configured to deliver flow to the nasal prongs from two directions via two connecting tubes which converged at a small diameter reservoir from which the prongs emerged, while the large bore cannula was configured to deliver flow to the nasal prongs from one direction using a larger diameter connecting tube. 
based on information consisting of only one pressure, one velocity, one temperature, etc., for each control volume, so it would be inconsistent to use much more information to define the geometry. Thus, the FAVOR $^{\text {mix }}$ technique retains the simplicity of rectangular elements while representing complex geometric shapes at a level consistent with the use of averaged flow quantities within each volume element. The simulation was performed with an eight-processor computer. The twoequation k-epsilon turbulent model was used to calculate the turbulence characteristics of the flow in incompressible conditions.

The generalized minimal residual (GMRES) method was used for pressure calculation from the linear system of continuity equations throughout the domain. Using GMRES, the system of equations is solved simultaneously throughout the domain by an iterative technique.

The simulation was carried out in two stages:

Steady state flow calculation (stage 1): In the first stage of the simulation, a steady state solution was obtained by solving the continuity, momentum and turbulence model equations for each cell of the computational mesh until a fully converged solution was obtained.

Particle injection and purge time calculation (stage 2): After a steady state solution was achieved, the velocity field was "frozen" by disabling the continuity, momentum, and turbulence equations. Then, a set of marker (virtual) particles was injected at the flow inlet. The particles were tracked until sufficient time was passed during which 99.9\% of the particles left the domain. Using a detailed post-processing procedure, the residence time distribution of the particles was calculated at 0.2 second time intervals. The normalized curves illustrating the incremental and cumulative fractions of residence time distribution versus particle residence time were plotted.

\section{Results}

The steady state flow modeling demonstrated a grossly similar pattern of flow behavior with both cannula configurations. The images in Figure 2 show the flow patterning, by depicting travel time, between the small bore nasal prong cannula design and the large bore nasal prong cannula design. In each nasal cavity, separated by the nasal septum and supplied by an individual nasal cannula prong, upper and lower vortices were formed around a central inward flow stream. The flow in these individual nasal cavities converged in the nasopharynx to create one flow path exiting through the oral cavity. A noticeable exception between the cannula configurations is that the small prong cannula resulted in a third, smaller and anterior vortex on each side of the nasal septum.

In the CFD figures presented in this paper, the flow pattern from the small prong cannula is shown in only one of the two nasal cavities for simplicity. The model was run using both nasal prongs, but the flow from each prong was by design identical given the configuration of the cannula. However, because the larger prong cannula design had flow reaching the cannula prongs from only one direction, flow patterns are shown on both sides of the nasal septum to elucidate dissimilarities resulting from differing flow rates between the two cannula prongs.

\section{Velocity vectors and velocity magnitude}

The velocity vectors are depictions that demonstrate the directionality of flow in the cavity. As again confirmed in Figure 3, both cannula designs demonstrate the same general pattern of flow, with a superior and inferior vortex generation. A notable difference was that the small anterior vortex seen with the small bore nasal prongs was associated with a slight entrainment of room air through the nose around the cannula prongs. Conversely, the large bore nasal prongs were shown to generate a slight retrograde nasal flow, exiting around the outside of the prongs. Velocity magnitude, presented in Figure 3 as in $\cdot \sec ^{-1}$, was greater around the vortices with the smaller prong cannula.

\section{Strain rate and turbulent kinetic energy}

The profiles for strain rate and turbulent kinetic energy look very similar throughout the nasal cavity for each cannula design. Strain rate, plotted in reciprocal of seconds $\left(\mathrm{sec}^{-1}\right)$, can be multiplied by viscosity to give shear stress, representing the jetting forces on the nasal walls. Because viscosity is consistent throughout, stain rate alone is sufficient for this analysis.

As shown in Figure 4, with the small bore cannula the strain rate is absorbed within the gas layers and does not impact the walls of the nasal cavity. The strain rate resulting from the large bore cannula tip does impact the nasopharyngeal walls, presumably a function of the wider jet stream having a closer proximity. Nonetheless, the strain was marginal.
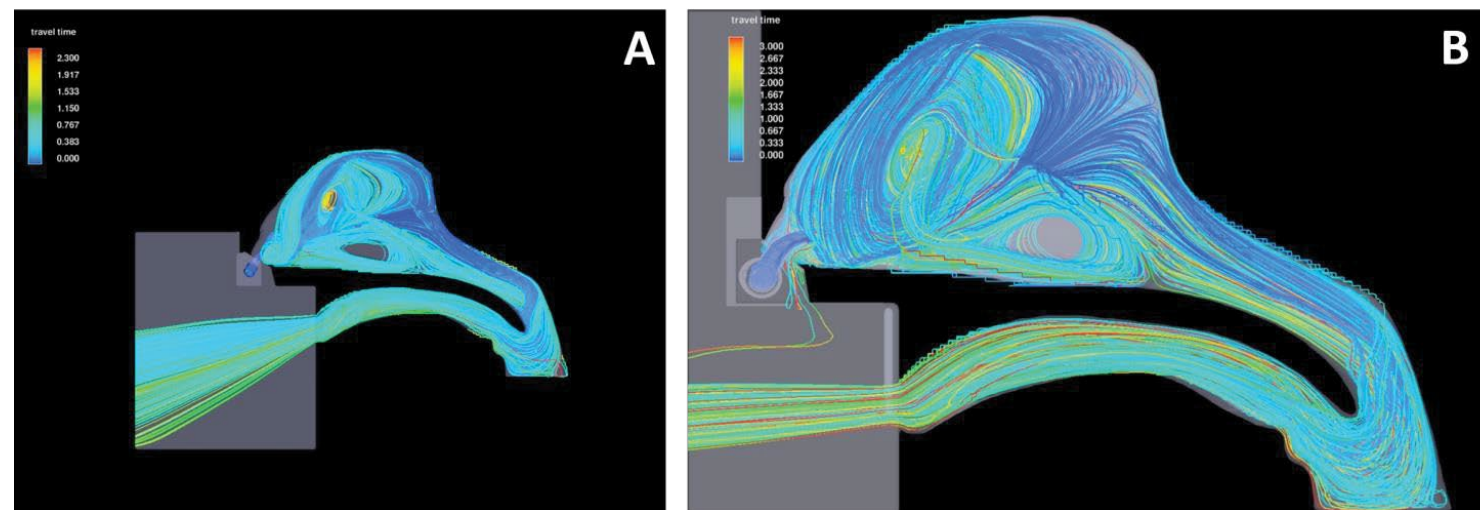

Figure 2: Flow Patterning and Travel Time. These flow modeling output frames represent the flow path and travel time for the small bore nasal prong cannula configuration $(A)$ and the larger bore prong configuration $(B)$. A cluster of particles was sent through the system and the color intensity represents the duration of time that the particles remain in the system. The smaller prong configuration was associated with the particles passing through more rapidly, spending less time in the system. Both cannulae resulted in a flow pattern with superior and inferior vortices encapsulating a central inward flow path on each side of the nasal septum, although the small bore cannula created a third vortex anteriorly. 
Citation: Miller TL, Saberi B, Saberi S (2016) Computational Fluid Dynamics Modeling of Extrathoracic Airway Flush: Evaluation of High Flow Nasal Cannula Design Elements. J Pulm Respir Med 6: 376. doi: 10.4172/2161-105X.1000376
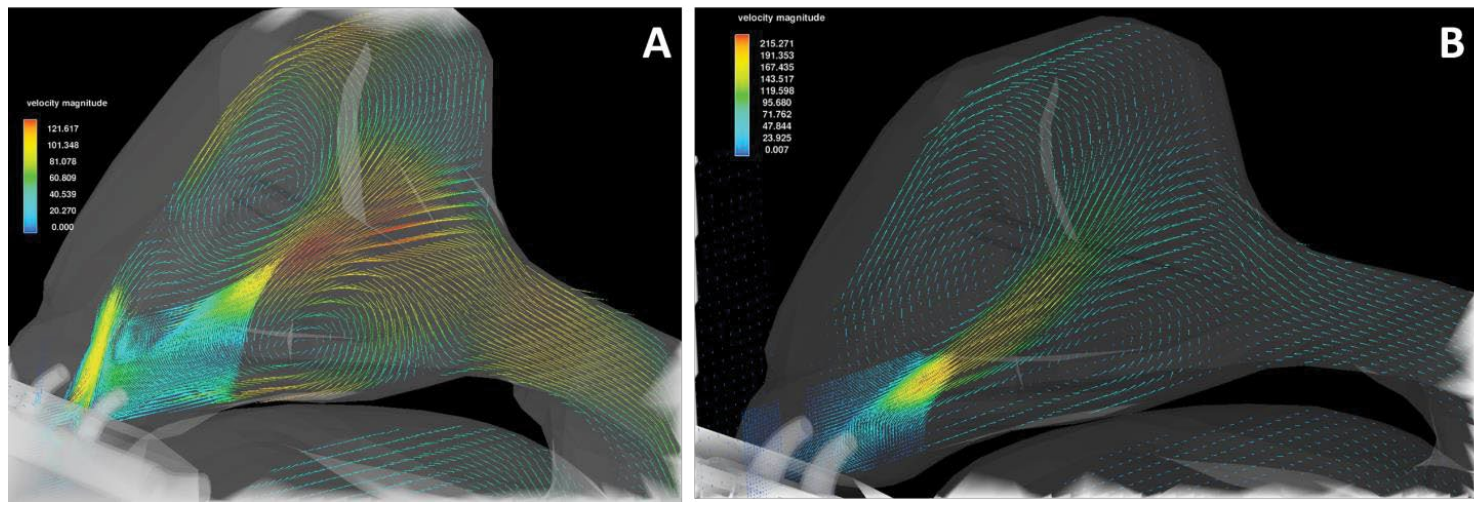

Figure 3: Velocity Vectors and Velocity Magnitude. The velocity vectors for the small bore nasal prong cannula configuration (A) and the larger bore prong configuration (B) demonstrate the directionality and relative magnitude $\left(\mathrm{In} \cdot \mathrm{Sec}^{-1}\right)$ of flow. Velocity Magnitude was greater around the vortices with the smaller prong cannula resulting in an addition anterior vortex on each side of the nasal septum. The small bore prongs also resulted in a slight entrainment of room air around the cannula prongs, while the larger nasal prongs generated a slight retrograde nasal flow exiting around the outside of the prongs.
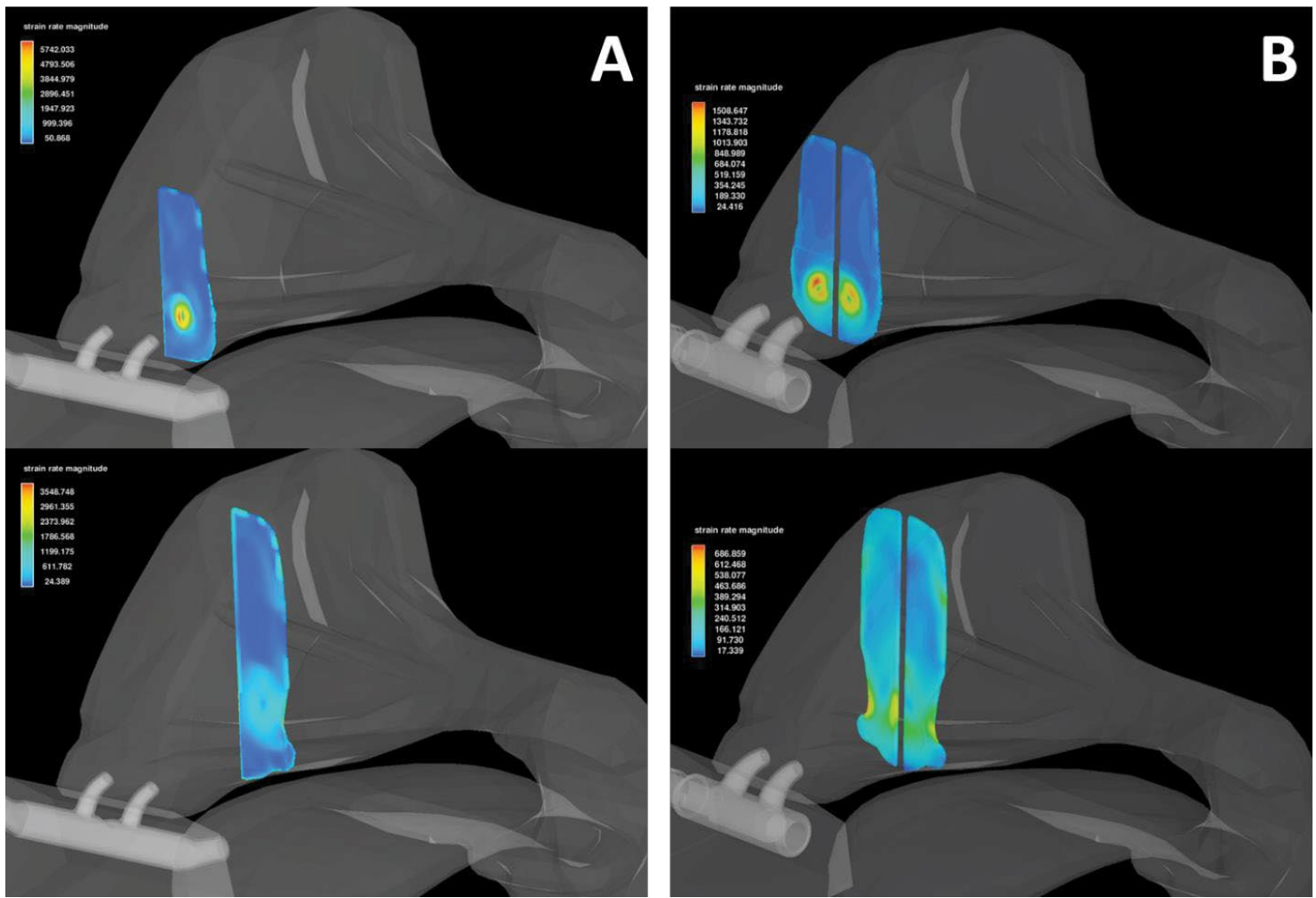

Figure 4: Strain Rate. Strain rate $\left(\mathrm{Sec}^{-1}\right)$ is show in two plates moving from the anterior region of the nasal cavity (top) to the mid-section of the nasal cavity (bottom). Strain rate, from which shear force on the tissues can be derived, only minimally impacts the walls of the nasal cavity with the small bore nasal prong cannula configuration (A). However, the strain rate resulting from the large bore cannula (B) does impact the nasopharyngeal walls. Because the large bore cannula design had flow delivered to the cannula prongs from only one direction, flow patterns are shown on both sides of the nasal septum to reveal any dissimilarity between the two prongs.

\section{Pressure contours}

As shown in Figure 5, CFD modeling demonstrates that pressure is not consistent throughout the airway or within any one region of the airway with HFNC application. Pressure is greater in every region of the nasal cavity with the large bore prongs, and the single sided feed to the large bore prong configuration resulted in a slight imbalance in flow between the two sides of the nasal cavity (disparity between the prongs) that is most notable in the pressure presentation.

Figure 5 shows the contours for airway pressure. The graph presents values for the largest area of uniform pressure in each CFD output region, moving anterior to posterior-inferior. Pressure associated with the small bore cannula ranged from a low of $-133 \mathrm{gf} \cdot \mathrm{sq}^{-1}(-21$ 


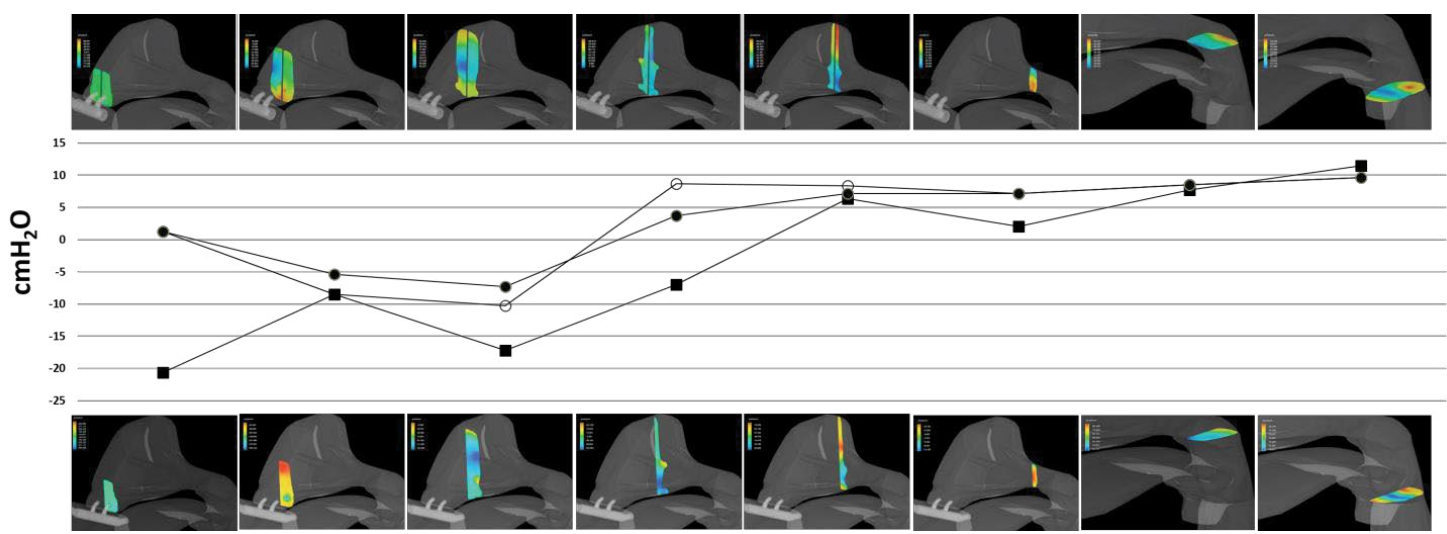

Figure 5: Pressure Contours. Pressure in the nasal / nasopharyngeal cavities are shown as flow modeling output frames moving from anterior to posterior / inferior (left to right) and presented graphically as the pressure for the largest area in each plate $\left(\mathrm{cmH}_{2} \mathrm{O}\right)$. The small bore nasal prong cannula configuration (bottom; squares) is compared to the large bore prong configuration (top; circles). The unilateral connecting tube design feeding the large bore cannula configuration resulted in a slight disparity in pressure contour between the two sides of the nasal septum; the right (proximal) side is represented by open circles, and the left (distal) side by closed circles. The colors indicate intensity of pressure, but the color palate is not consistent from image to image. Pressure here is reported as gf.Sq $\mathrm{In}^{-1}$, which is converted to $\mathrm{cmH}_{2} \mathrm{O}$ through multiplying by 0.155 .
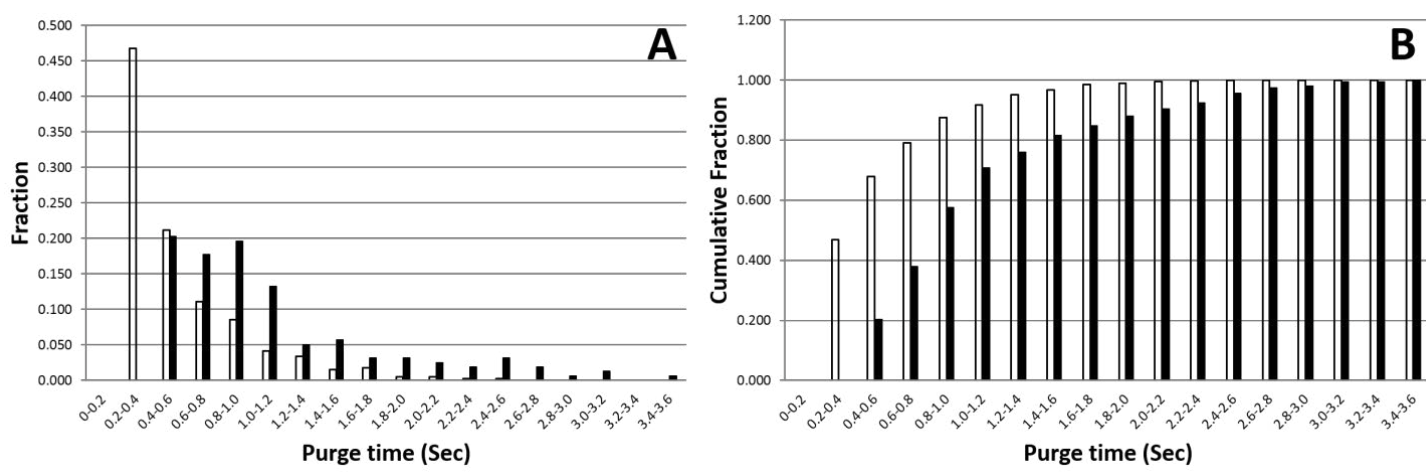

Figure 6: Purge Time. Results of the purge time assessment are presented as the fraction of injected particles that emerge from the system (through the oral cavity) within each 0.2 -second interval of time (A) and the cumulative fraction of particles that have passed through the system at the end of each time interval (B). The small prong cannula design (open bars) is compared to the large prong cannula design (closed bars). The small prong cannula resulted in a faster purge of the nasal, oral and pharyngeal cavities.

$\mathrm{cmH}_{2} \mathrm{O}$ ) just inside the nares, followed by a transient upward deflection associated with the anterior vortex, before rising in the pharyngeal region to +74 gf.sq $\mathrm{in}^{-1}\left(+11 \mathrm{cmH}_{2} \mathrm{O}\right)$ in the laryngopharynx. For the large bore cannula, pressure ranged from $-66 \mathrm{gf} \cdot \mathrm{sq} \mathrm{in} \mathrm{in}^{-1}\left(-10 \mathrm{cmH}_{2} \mathrm{O}\right)$ in the right-side mid-nasal region (proximal to the connecting tube) to +62 gf.sq in ${ }^{-1}\left(+10 \mathrm{cmH}_{2} \mathrm{O}\right)$ in the laryngopharynx. The smaller prong cannula resulted in a negative pressure value for most of the nasal cavity, becoming positive posteriorly where the flow converges in the pharyngeal region. Hence, with the small bore prongs air is entrained through the nose contributing to a slightly greater bulk flow through the airway. Pressure is positive at the nares with the larger prongs, resulting in a slight retrograde flow through the nose.

\section{Particle injection, travel time and purge time calculation}

The images in Figure 2 also depict flow travel time between the small bore nasal prong cannula design (Figure 2A) and the large bore nasal prong cannula design (Figure $2 \mathrm{~B}$ ). The color intensity represents the time that each particle is in the system, and the greater color array associated with the large bore prong cannula design indicates that the particles are taking longer to pass through the system.
Results of the purge time assessment are shown in Figure 6. The small bore cannula design resulted in almost one-half of the particles passing through the system in the first 0.4 seconds, whereas particles from the large bore cannula design did not start to emerge until after the first 0.4 second period. The small bore cannula design reached 99.9\% particle emission by $2.2 \mathrm{sec}$ and the large bore cannula design did not accomplish this milestone until $3.6 \mathrm{sec}$, taking $64 \%$ longer to purge the cavity of the particles.

\section{Discussion}

The goal of the project was to create a CFD model to evaluate the performance of two nasal cannula designs for HFNC therapy. The hypothesis was driven by pre-clinical animal studies that suggest that cannula design, primarily focused on nasal occlusion related to outer diameter of the prong, can dramatically impact effectiveness of purging extrathoracic dead space [6]. The current CFD data agree with the findings of Frizzola et al. [6] that a less occlusive prong facilitates this purge action. However, the current findings reveal that the impact is more related to dynamic energy induced by the greater velocity from the tighter prong nozzle as opposed to the lesser occlusion of the nares. 
The clinical approach for dead space purge techniques originates with tracheal gas insufflation within an endotracheal tube to wash $\mathrm{CO}_{2}$ rich gas from the airway during exhalation [17]. Conceptually, there is a finite amount of time (late stage exhalation and endexpiratory pause) to purge the space, and a finite amount of dead space volume that can be purged. As flow is increased, more of the volume can be purged until flow is sufficient to purge all of the volume in the allotted time, after which additional flow produces no additional effect. Frizzola et al. [6] expanded this concept to purging the extrathoracic dead space with a nasal cannula, showing that a single prong cannula design that occluded no more than $50 \%$ of the nares was more effective at a lower flow, and with lower resulting airway pressure, compared to a more occlusive prong design [6]. This single prong cannula design also produced two-fold the velocity at the cannula tip, compared to the conventional 2 prong cannula, at each matched flow rate.

The two cannula designs tested here were the commercially available Vapotherm regular adult cannula (Vapotherm Exeter, $\mathrm{NH}$, USA; the smaller prong cannula), and a larger bore cannula design that was conceived by Vapotherm but never made commercially available pursuant to these data. Note that this larger bore cannula design is not as large as some cannulae that have been commercially available with other HFNC systems. A flow rate of $20 \mathrm{~L} \cdot \mathrm{min}^{-1}$ was chosen for the model because this flow rate should be low enough to avoid saturating the purge effect and allow resolution to differentiate between the cannula purge times. This determination of flow rate was derived by extrapolating a minimum HFNC flow rate from the standard formula for predicting effective inspired oxygen fraction from a low flow oxygen cannula [18]. This extrapolation defines the flow rate where an oxygen cannula (delivering pure oxygen) would result in an effective inspiratory oxygen fraction of 1.0 , as being $25 \mathrm{~L} \cdot \mathrm{min}^{-1}$. The geometry for airway anatomy used in this model was originally derived from a human scan, and therefore proportionally correct.

The basic flow patterns were similar for both cannula designs, encapsulating a central inward flow path between upper and lower vortices on each side of the nasal septum. However, the time required to purge the nasal cavity was divulged as a function of flow energy to clear these vortices, and this is where a divergence between cannula performances was noted. Gas flow velocity was greater around the vortices with the small cannula, resulting in an addition anterior vortex. This is a function of the greater velocity from the tip of the smaller cannula; i.e., the same flow in L- $\mathrm{min}^{-1}$ being delivered through a smaller cross-sectional area equates to a greater velocity. There was also a slightly greater bulk flow with the smaller prong cannula related to the slight entrainment air, versus the retrograde nasal flow seen with the larger prong cannula. This minor entrainment versus retrograde nasal flow seen with the two cannula designs would not result in a meaningfully dilution of the cannula delivered gas, but does underscore a marked change in flow dynamics between cannula designs.

An important question in the analysis for strain rate that pertains to tissue shear forces, is whether the jetting effect from the cannula tip resulted in undue shear on the airway mucosal tissue. While both cannula designs showed a similar strain rate, most of the strain was absorbed between gas laminae. This projection of elevated strain on the mucosa seen only with the large bore cannula is presumably a function of the closer proximity to the wall. Regardless, even in the case of the large bore cannula, shear does not appear to be of a sufficient magnitude to result in acute damage.

The question of airway pressure generation is a common concern among users of HFNC. Interestingly, these data show that pressure is negative, albeit slightly, throughout most of the nasal cavity with the small prong cannula, becoming positive posteriorly where the flow streams converge in the pharynx. Pressure is greater in every region of the airway with the larger prong cannula, resulting in a positive pressure in the most anterior region of the nasal cavity, and thus retrograde flow through the nose. The negative pressure in the anterior region of the nasal cavity seen with the smaller prongs resulted in the slight entrainment of air. The mild positive pressure noted in the pharyngeal region would be diminished during an inhalation by the patient, but apply mild expiratory resistance similar to the pursed lip breathing maneuver used by some chronic respiratory patients to control shortness of breath [19].

The CFD model used was static in that breathing (inhalation/ exhalation) was not simulated within anatomical geometry. The rationale for using the static model was the primary objective of measuring time to purge the extrathoracic dead space, which would then be reset with each patient breath. By not accounting for breathing, the model was able to quantify purge times longer in duration than the respiratory cycle.

The smaller prong cannula design cleared the extrathoracic dead space within 2.2 seconds, whereas the larger prong design required 3.6 seconds. These values for purge time are very meaningful relative to human physiology, given that the expiratory period in a normally breathing adult would be 3.3 seconds (considering 12 breaths per minute and an inspiratory time fraction of 0.33 ), and would be less in an adult experiencing respiratory distress as breathing frequency increases. Therefore, at the flow rate of $20 \mathrm{~L} \cdot \mathrm{min}^{-1}$, the small prong cannula design would have been able to purge the extrathoracic dead space between breaths. Conversely, the large bore cannula design would not be as effective and would require additional flow to accomplish the same objective. These data also support the supposition introduced in the Methods section that $25 \mathrm{~L} \cdot \mathrm{min}^{-1}$ would be a starting point for HFNC therapy in a distressed adult.

\section{Limitations}

This model is computational for one set of airway geometries and cannula positions. Actual variations from person to person with regard to airway geometry, as well as cannula positioning, may influence the reported flow parameters. This study used a static model that did not account for breathing, and while chosen for reasons discussed herein, did not account for expiratory flow from the lungs.

\section{Conclusions}

The CFD model developed for this project was useful in characterizing flow patterns and purge time with two cannula designs for HFNC administration. Moreover, this model can support development of advanced cannula configurations, and herein defined flow velocity as a primary factor for accomplishing purge of the upper airway. Herein, the smaller prong configuration resulted in a faster purge of extrathoracic dead space for a given flow, compared to the larger prong configuration $(28 \%$ faster at $20 \mathrm{lpm})$. Therefore, the smaller bore prongs can accomplish the objective of HFNC therapy (i.e., complete extrathoracic dead space purge within the window of time between breaths) at a much lower flow rate than the larger bore prongs. These data are in agreement with the results of the physiologic research indicating that smaller prongs give better efficacy at a lower flow rate with lower resultant airway pressure. However, the current findings suggest that this difference is related to dynamic energy induced by the greater velocity from the tighter prong nozzle, as opposed to a lesser occlusion of the nares. 


\section{Acknowledgement}

This study was sponsored by Vapotherm Inc., Exeter, NH, USA, and the work was performed at Green Twirl Energy, Ltd., Ontario, Canada.

\section{References}

1. Dysart K, Miller TL, Wolfson MR, Shaffer TH (2009) Research in high flow therapy: mechanisms of action. Respir Med 103: 1400-1405.

2. Gotera C, Lobato DS, Pinto T, Winck JC (2013) Clinical evidence on high flow oxygen therapy and active humidification in adults. Rev Port Pneumol 19: 217-227.

3. Kelly MG, McGarvey LP, Heaney LG, Elborn JS (2001) Nasal septal perforation and oxygen cannulae. Hosp Med 62: 248.

4. Robertson NJ, McCarthy LS, Hamilton PA, Moss AL (1996) Nasal deformities resulting from flow driver continuous positive airway pressure. Arch Dis Child Fetal Neonatal Ed 75: F209-F212.

5. Woodhead DD, Lambert DK, Clark JM, Christensen RD (2006) Comparing two methods of delivering high-flow gas therapy by nasal cannula following endotracheal extubation: a prospective, randomized, masked, crossover trial. J Perinatol 26: 481-485.

6. Frizzola M, Miller TL, Rodriguez ME, Zhu Y, Rojas J, et al. (2011) High-flow nasal cannula: impact on oxygenation and ventilation in an acute lung injury model. Pediatr Pulmonol 46: 67-74.

7. Dewan NA, Bell CW (1994) Effect of low flow and high flow oxygen delivery on exercise tolerance and sensation of dyspnea. A study comparing the transtracheal catheter and nasal prongs. Chest 105: 1061-1065.

8. Greenspan JS, Wolfson MR, Shaffer TH (1991) Airway responsiveness to low inspired gas temperature in preterm neonates. J Pediatr 118: 443-445.

9. Spence KL, Murphy D, Kilian C, McGonigle R, Kilani RA (2007) High-flow nasal cannula as a device to provide continuous positive airway pressure in infants. J Perinatol 27: 772-775.
10. Parke R, McGuinness S, Eccleston M (2009) Nasal high-flow therapy delivers low level positive airway pressure. $\mathrm{Br} \mathrm{J}$ Anaesth 103: 886-890.

11. Spentzas T, Minarik M, Patters AB, Vinson B, Stidham G (2009) Children with respiratory distress treated with high-flow nasal cannula. J Intensive Care Med 24: 323-328.

12. Hasani A, Chapman TH, McCool D, Smith RE, Dilworth JP, et al. (2008) Domiciliary humidification improves lung mucociliary clearance in patients with bronchiectasis. Chron Respir Dis 5: 81-86.

13. Rea H, McAuley S, Jayaram L, Garrett J, Hockey H, et al. (2010) The clinical utility of long-term humidification therapy in chronic airway disease. Respir Med 104: 525-533.

14. Danan C, Dassieu G, Janaud JC, Brochard L (1996) Efficacy of dead-space washout in mechanically ventilated premature newborns. Am J Respir Crit Care Med 153: 1571-1576.

15. Dassieu G, Brochard L, Agudze E, Patkai J, Janaud JC, et al. (1998) Continuous tracheal gas insufflation enables a volume reduction strategy in hyaline membrane disease: technical aspects and clinical results. Intensive Care Med 24: 1076-1082.

16. Benditt J, Pollock M, Roa J, Celli B (1993) Transtracheal delivery of gas decreases the oxygen cost of breathing. Am Rev Respir Dis 147: 1207-1210.

17. Miller TL, Blackson TJ, Shaffer TH, Touch SM (2004) Tracheal gas insufflationaugmented continuous positive airway pressure in a spontaneously breathing model of neonatal respiratory distress. Pediatr Pulmonol 38: 386-395.

18. Cairo JM, Pilbeam SP (2004) Mosby's Respiratory Care Equipment. (7th edn), St Louis: Elsevier Mosby, Missouri, USA.

19. Borge CR, Hagen KB, Mengshoel AM, Omenaas E, Moum T, et al. (2014) Effects of controlled breathing exercises and respiratory muscle training in people with chronic obstructive pulmonary disease: results from evaluating the quality of evidence in systematic reviews. BMC Pulm Med 14: 184. 